\title{
Phosphate binding therapy in dialysis patients: focus on lanthanum carbonate
}

\author{
Ismail A Mohammed \\ Alastair J Hutchison
}

Manchester Institute of Nephrology and Transplantation, Manchester Royal Infirmary, Oxford Road, Manchester, UK
Correspondence: Alastair J Hutchison

Clinical Director Renal Services, Manchester Institute of Nephrology and Transplantation, Manchester Royal Infirmary, Oxford Road, Manchester, UK Tel +44 I6I 2764488

Fax +44 I6I 2768022

Email alastair.hutchison@CMMC.nhs.uk

\begin{abstract}
Hyperphosphatemia is an inevitable consequence of end stage chronic kidney disease and is present in the majority of dialysis patients. Recent observational data has associated hyperphosphatemia with increased cardiovascular mortality among dialysis patients. Dietary restriction of phosphate and current dialysis prescription practices are not enough to maintain serum phosphate levels within the recommended range so that the majority of dialysis patients require oral phosphate binders. Unfortunately, conventional phosphate binders are not reliably effective and are associated with a range of limitations and side effects. Aluminium-containing agents are highly efficient but no longer widely used because of well established and proven toxicity. Calcium based salts are inexpensive, effective and most widely used but there is now concern about their association with hypercalcemia and vascular calcification. Sevelamer hydrochloride is associated with fewer adverse effects, but a large pill burden and high cost are limiting factors to its wider use. In addition, the efficacy of sevelamer as a monotherapy in lowering phosphate to target levels in severe hyperphosphatemia remains debatable. Lanthanum carbonate is a promising new non-aluminium, calcium-free phosphate binder. Preclinical and clinical studies have demonstrated a good safety profile, and it appears well tolerated and effective in reducing phosphate levels in dialysis patients. Its identified adverse events are apparently mild to moderate in severity and mostly GI related. It appears to be effective as a monotherapy, with a reduced pill burden, but like sevelamer, it is significantly more expensive than calcium-based binders. Data on its safety profile over 6 years of treatment are now available.
\end{abstract}

Keywords: hyperphosphatemia, lanthanum carbonate, dialysis, phosphate binding

\section{Introduction}

Hyperphosphatemia is an almost inevitable consequence of chronic kidney disease (CKD). It occurs in the majority of dialysis patients and continues to represent a major challenge to clinical nephrologists. Indeed more than $36 \%$ of UK haemodialysis patients have plasma phosphate of $\geq 1.8 \mathrm{mmol} / \mathrm{L}(5.5 \mathrm{mg} / \mathrm{dL})$ despite dietary manipulation and prescription of oral phosphate binders (Lamb et al 2007).

Normal kidneys filter large amounts of organic phosphate of which more than $90 \%$ is reabsorbed by the renal tubules. Early renal dysfunction reduces filtered phosphate but also decreases tubular reabsorption, so that the urinary phosphate excretion continues to match gastrointestinal (GI) absorption. Consequently, the net balance between phosphate input and output is maintained for a period of time with only little change in serum phosphate levels. However, as renal function deteriorates further, this homeostatic mechanism fails resulting in positive phosphate balance and progressive hyperphosphatemia.

Untreated hyperphosphatemia can lead to secondary hyperparathyroidism (SHPT), renal osteodystrophy, vascular calcification and increased morbidity and mortality (Lowrie and New 1990; Delmez and Slatopolsky 1992; Block et al 2004). Retrospective cross-sectional studies suggest that a serum phosphate greater 
than $6.5 \mathrm{mg} / \mathrm{dL}(2.10 \mathrm{mmol} / \mathrm{L})$ is associated with a $27 \%$ higher mortality risk (relative risk [RR] +1.27) compared with patients with a phosphate level of $2.4-6.5 \mathrm{mg} / \mathrm{dL}$ $(0.78-2.10 \mathrm{mmol} / \mathrm{L})$, and relative risk increases as serum phosphate rises (Block and Port 2000). Block et al found that a calcium $\times$ phosphate product above $72 \mathrm{mg}^{2} / \mathrm{dL}^{2}$ was also associated with a significantly higher relative risk of death $(\mathrm{RR}+1.34)$, but this is not surprising if the majority of the risk is attributed to the phosphate. Consequently, phosphate control remains an important therapeutic target in management of CKD, not only to halt progression to secondary hyperparathyroidism but also to reduce the risk of vascular calcification and cardiovascular mortality, although no prospective interventional studies currently exist to demonstrate that this is achievable.

Unfortunately phosphate control has not been significantly improved over the past two decades. Several factors may have contributed to this, including the difficulty of adhering to renal diets, phosphate binder prescriptions and inadequate phosphate clearance by dialysis. In addition, factors such as cost, tolerability, palatability, safety, and efficacy are also important (Table 1).

We review current management of hyperphosphatemia with a particular focus on the pharmacology, clinical efficacy, and safety profile of lanthanum for treatment of elevated serum phosphate in dialysis patients.

\section{Management of hyperphosphatemia in chronic kidney disease}

The standard approach to management of elevated serum phosphate in CKD includes dietary restriction, dialysis and drug treatment using oral phosphate binders. Unfortunately, there are many limitations to this approach which may explain the current inability to adequately treat hyperphosphatemia.

Firstly, dietary phosphate restriction is impractical for many patients who mostly eat pre-prepared, supermarket meals rather than freshly prepared foods as in the relatively recent past. In addition it can only be restricted to a certain

Table I Suggested characteristics of an ideal oral phosphate binder High affinity for binding phosphate - low dose (pill burden) required Rapid phosphate binding regardless of ambient $\mathrm{pH}$

Low solubility

Low systemic absorption (preferably none)

Non toxic and without side effects

Solid oral dose form

Palatable - encourages compliance

Inexpensive extent without risking protein malnutrition, particularly in elderly patients (Kopple and Coburn 1973).

Secondly, conventional thrice-weekly, 4-hour hemodialysis removes approximately $1000 \mathrm{mg}$ of phosphate each treatment, but this is generally insufficient to maintain phosphate levels within the recommended targets even if oral phosphate intake is significantly restricted. Peritoneal dialysis is little better in this respect (Delmez et al 1982). Kinetic studies of hemodialysis have shown that since phosphate is predominantly intracellular, serum levels drop rapidly in the first 1-2 hours of dialysis and then reach a plateau. They then rise relatively quickly in the first few hours after termination of dialysis, the so-called 'rebound phenomenon'. Although short daily and slow nocturnal haemodialysis may be effective in reducing serum phosphate levels, logistic, cost and patient acceptance issues limit widespread usage of such modalities (Lowrie and Lew 1990). Thus around 90\% of dialysis patients continue to need additional therapeutic maneuvres to improve their phosphate levels.

Traditionally available oral phosphate binding agents, though effective in lowering serum phosphate levels are not ideal and most have limitations of one sort or another (Table 2). Aluminium-based phosphate binders are highly efficient but have been associated with cognitive disturbances, osteomalacia, and anemia which restrict use (Wills and Savory 1983; Gonzalez-Revalderia et al 2000). No safe dose of aluminium has been identified and dialysis patients who take it even in modest doses have been reported to develop clinical evidence of toxicity (Malluche 2002). Nonetheless aluminium salts are still used as short term 'salvage' therapy to achieve acute control of high phosphate levels, and are also used in patients whose prognosis is felt to be so short, because of other co-morbidities, that the advantages may outweigh the risks.

Calcium-based binders (acetate and carbonate) are effective and inexpensive but their prolonged administration can result in hypercalcemia in over $50 \%$ of patients, especially when administered with vitamin D analogues (Schaefer et al 1992). In addition they can result in oversuppression of parathyroid hormone (PTH), adynamic bone, and are associated with both soft tissue and vascular calcification (Goodman et al 2000; Goodman 2001). However, it is worth remembering that vascular calcification was first seen in the 1980s when aluminium hydroxide was the only commonly available phosphate binder. Several investigators have found calcium acetate to be more effective in binding intestinal phosphate, per mmol of administered elemental calcium, than calcium carbonate (Mai et al 1989). However, 
Table 2 Comparison of currently available oral phosphate binders

\begin{tabular}{|c|c|c|}
\hline Phosphate binder & Advantages & Disadvantages \\
\hline \multirow[t]{5}{*}{ Calcium carbonate } & Aluminium free & Efficacy influenced by $\mathrm{pH}$ \\
\hline & Moderately effective & Unpalatable \\
\hline & Moderate pill burden & Hypercalcemia \\
\hline & Cheap & GI side effects \\
\hline & & Possible ectopic calcification \\
\hline \multirow[t]{5}{*}{ Calcium acetate } & Aluminium free & Large tablets need to be swallowed \\
\hline & Efficacy some what $\mathrm{pH}$ dependent & Hypercalcemia \\
\hline & Moderately cheap & Gl side effects \\
\hline & Lower calcium load than carbonate & Possible ectopic calcification \\
\hline & Calcium free & \\
\hline \multirow[t]{3}{*}{ Aluminium salts } & High efficacy regardless of $\mathrm{pH}$ & Aluminium toxicity \\
\hline & Cheap & No definite safe dose \\
\hline & Not pH dependent & Frequent monitoring needed \\
\hline \multirow[t]{4}{*}{ Magnesium salts } & Moderate pill burden & GI side effects \\
\hline & Calcium and aluminium free & Not widely used \\
\hline & Moderate efficacy & Magnesium monitoring \\
\hline & Moderate pill burden & \\
\hline \multirow[t]{5}{*}{ Sevelamer } & Calcium and aluminium free & Expensive \\
\hline & No Gl tract absorption & Efficacy influenced by $\mathrm{pH}$ \\
\hline & Moderate efficacy & High pill burden \\
\hline & Reduces total and LDL cholesterol & GI side effects \\
\hline & & Binds fat-soluble vitamins \\
\hline \multirow[t]{4}{*}{ Lanthanum carbonate } & Calcium and aluminium free & Expensive \\
\hline & Chewed, not swallowed whole & Gl side effects \\
\hline & High efficacy regardless of $\mathrm{pH}$ & Minimal Gl absorption \\
\hline & Low pill burden & \\
\hline
\end{tabular}

Abbreviations: GI, gastrointestinal; LDL cholesterol, low density lipoprotein cholesterol.

compliance and patient tolerability are generally poor with calcium acetate and the studies showing a greater reduction in mean serum phosphate level, compared with the same dose of calcium carbonate, were relatively short-term (Qunibi et al 2004).

Magnesium-containing phosphate binders can be used as an alternative to calcium-based agents but generally they are less effective, and are associated with increased serum magnesium levels and diarrhea. However, magnesium iron hydroxycarbonate is a new oral phosphate binder currently undergoing clinical trials, with promising results presented in abstract form only so far (McIntyre 2007).

Sevelamer hydrochloride was the first synthetic nonaluminium and calcium-free phosphate binder to become available. It was originally developed to lower plasma lipids and has this beneficial side effect in CKD patients. In several open label studies sevelamer appears as effective as calcium-based binders in lowering phosphate, but without the tendency to promote hypercalcemia (Bleyer et al 1999). Furthermore, there is some evidence that sevelamer hydrochloride can attenuate coronary and aortic calcification compared with calcium-based phosphate binders (Chertow et al 2002). Despite these advantages, GI disturbances, metabolic acidosis, and cost are limiting factors affecting the wider use of sevelamer hydrochloride. Unfortunately the large pill burden required to achieve target phosphate levels can adversely affect patient adherence. Nonetheless, sevelamer remains an important current therapy in the management of hyperphosphatemia. More recently lanthanum carbonate became widely available in the US (January 2005) and EU (July 2006), and is also a nonaluminium-, noncalcium-based binder.

\section{Pharmacology}

Lanthanum is a naturally occurring rare-earth element with a molecular weight of $139 \mathrm{Da}$ and atomic number 57. The element was discovered by Carl Gustaf Mosander in 1839. 
It is particularly abundant in China where it is mined, but can be found in many green leafy vegetables and also in tap water in the UK. As a phosphate binder, lanthanum is ingested as the carbonate salt, and it dissociates in the upper GI tract to the lanthanum ion $\left(\mathrm{La}^{3+}\right)$. It binds phosphate ionically, optimally at $\mathrm{pH} 3-5$, while retaining its phosphate-binding capacity across the full $\mathrm{pH}$ range from 1 to 7 (Hutchison and Al-Baaj 2005). Unlike calcium-based binders and sevelamer hydrochloride, lanthanum has been shown in vitro to bind phosphate efficiently even at the low $\mathrm{pH}$ found in the stomach, as well as the high $\mathrm{pH}$ values found in the duodenum and jejunum. This range of $\mathrm{pH}$ for binding phosphate is similar to that seen with aluminium salts $(3-5)$.

Animal studies have shown that lanthanum has similar phosphate binding efficacy to aluminium, but dramatically lower oral bioavailability. In a nephrectomized rat model it reduced urinary phosphate levels to the same extent as aluminium, and more effectively than calcium carbonate or sevelamer (Finn et al 2004). Lanthanum has a low potential for accumulation with only $0.00005 \%$ of the oral dose being absorbed via the canine GI tract (Shire Pharmaceuticals Group, data on file) compared with $0.05 \%-0.1 \%$ for aluminium (Knoll et al 1984). The small absorbed fraction is eliminated primarily by the liver, not the kidneys, with $85 \%$ of this being eliminated in bile and 13\% directly across the gut wall (Hutchison 2004; Damment and Pennick 2007). In rats $99.3 \%$ of an oral dose is excreted in faeces with only $0.004 \%$ excreted in urine (Hutchison 2004). In man the absolute bioavailability of lanthanum (administered as lanthanum carbonate) was extremely low $(0.00127 \% \pm 0.00080 \%)$, with individual values in the range of $0.00015 \%-0.00224 \%$ (Pennick et al 2006). Renal clearance was negligible after oral administration (1.36 \pm $1.43 \mathrm{~mL} / \mathrm{min}$ ), and intravenous administration confirmed this low renal clearance $(0.95 \pm 0.60 \mathrm{~mL} / \mathrm{min})$ - just $1.7 \%$ of total plasma clearance. Furthermore, in contrast to aluminium, lanthanum does not cross the blood brain barrier, so that the potential for neurological adverse side effects is extremely low (Damment et al 2007). Lanthanum carbonate is not known to induce any systemic drug reactions and has no effect on cytochrome P450 enzymes.

\section{Clinical efficacy}

The results of published data on lanthanum carbonate demonstrate that lanthanum has many of the characteristics of an effective phosphate binder. Pre-clinical animal studies suggested that lanthanum may be similar to aluminium in phosphate lowering capacity but with a much more favourable safety profile.
Phase II studies revealed a statistically significant decrease in phosphate levels in patients receiving lanthanum carbonate at a dose of 1500-3000 mg/day in two double blind placebo controlled studies (Hutchison et al 2004; Al-Baaj et al 2005). The maximal decrease in serum phosphate levels occurred after 3 weeks of treatment and was maintained over the 3-6 weeks of treatment.

Three large multicenter phase III studies were performed to evaluate the efficacy and safety of lanthanum carbonate. The first trial was a 13-week randomized, double-blind, placebo-controlled, parallel-group study which showed a highly significant difference in the mean serum phosphate levels between lanthanum and placebo groups (Joy and Finn 2003). Serum phosphate levels were controlled at $<1.9 \mathrm{mmol} / \mathrm{L}$ in $59 \%$ of patients receiving lanthanum vs $23 \%$ in the placebo group. Moreover, $66 \%$ of the lanthanumtreated group maintained a controlled phosphate level at study end point, compared with $31 \%$ of the placebo group. $\mathrm{Ca} \times \mathrm{P}$ product $(52.37 \pm 14.89$ vs $66.59 \pm 18.30 \mathrm{mg} / \mathrm{dL}$; $\mathrm{p}<0.0001)$, and serum PTH levels (209.41 \pm 152.65 vs $291.80=194.82 \mathrm{pg} / \mathrm{mL}$; $\mathrm{p}<0.01$ ) were significantly lower with lanthanum carbonate treatment than with placebo.

In a second large prospective, randomized, European, multi-center, open label comparator trial, the efficacy of lanthanum carbonate was compared with calcium carbonate (Hutchison et al 2005). In total around 800 patients were randomized (533 to lanthanum and 267 to calcium carbonate). After 1-3 weeks of 'washout' from any previous binder therapy, patients with hyperphosphatemia (serum phosphate $>1.8 \mathrm{mmol} / \mathrm{L}(5.58 \mathrm{mg} / \mathrm{dL}))$ were randomized to 5 weeks of dose titration with either lanthanum carbonate (375-3000 mg/day) or calcium carbonate (1500-9000 mg/ day) followed by a 20 -week maintenance period. The primary end point was reduction of serum phosphate levels to $\leq 1.8 \mathrm{mmol} / \mathrm{L}$. The secondary efficacy parameter was maintenance of phosphate control at $<1.8 \mathrm{mmol} / \mathrm{L}$ for 6 months or longer. PTH, calcium, and calcium $\times$ phosphate product levels were also monitored throughout the study.

After 9 weeks of treatment both groups had serum phosphate levels of around $1.69 \mathrm{mmol} / \mathrm{L}$ (Figure 1). The proportion of patients achieving controlled phosphate levels of $<1.8 \mathrm{mmol} / \mathrm{L}$ was similar in both treated groups (lanthanum $65.8 \%$, calcium $63.9 \%$ ) at the end of maintenance phase $(\mathrm{p}=\mathrm{NS})$. The reduction in calcium $\times$ phosphate product was generally greater in the lanthanum carbonate group at the end of the maintenance phase $-1.59 \mathrm{vs}-1.2 \mathrm{mmol} / \mathrm{L}$ ). Perhaps most importantly from a clinical perspective, there was a significantly higher incidence of hypercalcemia in the calcium-treated group 


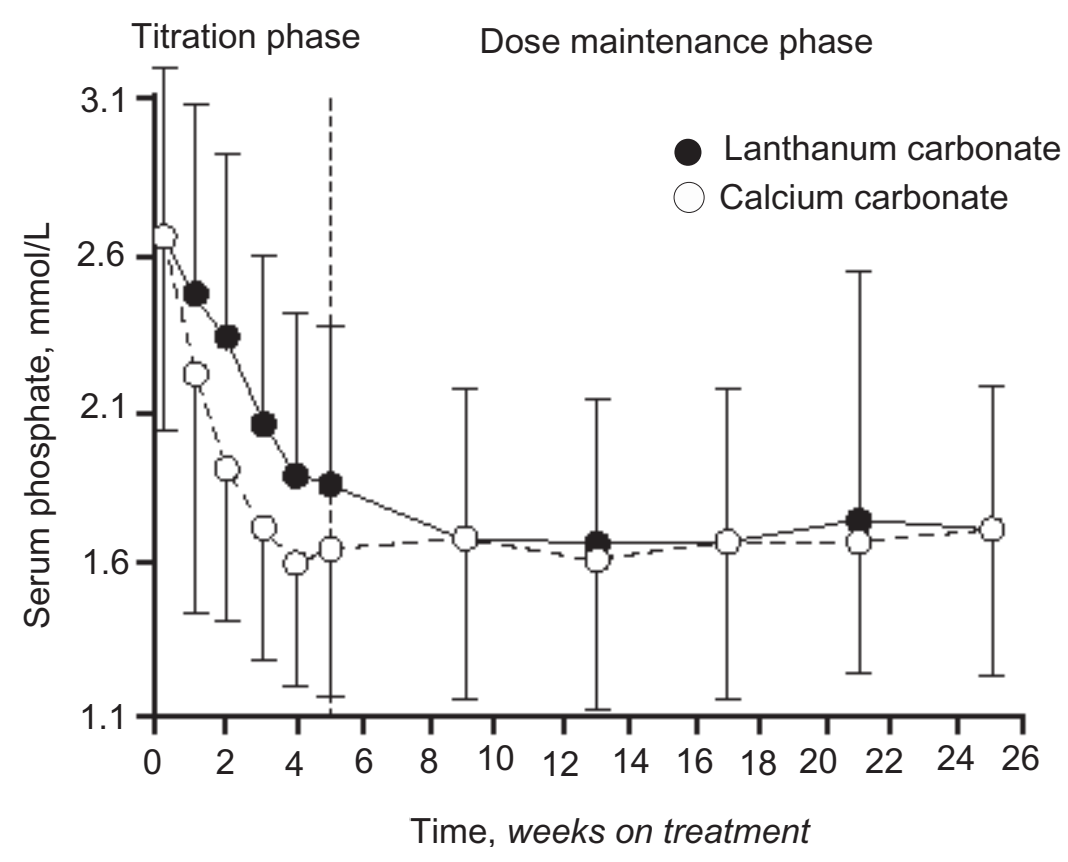

Figure I Mean serum phosphate levels during titration and maintenance treatment in the ITT population.

$(20.2 \%)$ than in the lanthanum group $(0.4 \%)$, as one would expect with a noncalcium-based binder.

A similar but longer-term study was performed in the US, in which lanthanum monotherapy was compared with any other standard phosphate binder or combination of binders (Finn 2006). After washout, patients were randomized to receive lanthanum carbonate $(n=682)$ or their pre-study phosphate binder $(n=677)$. Over a 6-week period, lanthanum carbonate was titrated to a maximum daily dose of $3000 \mathrm{mg}$ elemental lanthanum (serum phosphate target levels for titration were $\leq 1.90 \mathrm{mmol} / \mathrm{L}$ ). Over 2 years of follow up, phosphate control was similar in both groups but in the lanthanum group serum calcium was lower and serum PTH levels were maintained in the range recommended by K/DOQI. The most common adverse events were gastrointestinal. The incidences of events in the lanthanum and standard therapy groups were nausea, $37 \%$ vs $29 \%$; vomiting, $27 \%$ vs $22 \%$, and diarrhea ( $24 \%$ in each group). There was no indication of liver toxicity, suppression of erythropoiesis, or changes in the mini-mental state examination, and the authors concluded that the 2-year tolerability and efficacy of lanthanum were similar to those seen with any other standard therapy.

Subsequently the effect of lanthanum carbonate compared with calcium carbonate on development of renal osteodystrophy was evaluated in an open label bone biopsy study among patients undergoing dialysis (D'Haese et al 2003). At base line $98 \%$ of patients in each group had evidence of renal osteodystrophy in their bone biopsy.
Ninety-eight patients were randomized to receive lanthanum or calcium carbonate at a dose of 3750 or $9000 \mathrm{mg} /$ day respectively for 1 year, followed by a repeat bone biopsy. After 1 year of treatment, 63 paired bone biopsy samples were analyzable. A greater proportion of patients with low bone turnover lesions (adynamic bone or osteomalacia) at their baseline biopsy approached normalization on lanthanum treatment, compared to those given calcium (71\% vs $43 \%$ respectively). At the end of the study $30 \%(n=9)$ of calciumtreated patients had adynamic bone histology, compared with only $9 \%(n=3)$ of the lanthanum-treated group. Moreover, no evidence of any adverse effect on osteoblast function could be seen, and there was no correlation between bone lanthanum content and parathyroid hormone levels.

\section{Safety data}

Preclinical animal studies of lanthanum have shown no adverse effects at doses up to $2000 \mathrm{mg} / \mathrm{kg}$ body weight, suggesting a large safety margin for this compound. More importantly clinical studies have shown no evidence of toxicity affecting liver, bone or brain.

In common with all oral phosphate binders, lanthanum carbonate causes some GI side effects in around $20 \%$ of patients, but these seem to be relatively minor in most (abdominal pain, nausea, vomiting, diarrhea, and constipation). Avoidance of hypercalcemia is the major benefit of any noncalcium-based binder, and this may prove to be lanthanum's biggest safety benefit. 
Long-term safety of lanthanum has been examined in a number of open-label extension studies with exposure over 6 months (Hutchison et al 2005), 2 years (Finn 2006), 3 years (Hutchison et al 2006), and most recently 6 years (Hutchison et al 2008). In all these studies no evidence of toxicity has been seen, and in particular no adverse effects related to liver, bone, or brain, which have been often raised as possible target organs despite the paucity of supporting data.

In the large phase III prospective study of 197 patients randomized to receive either lanthanum carbonate or any other 'standard' phosphate binders, bone biopsies were obtained at baseline and again after either 1 or 2 years of treatment. No adverse effects on bone histology were seen in the lanthanum group. In another study 11 patients selected at random who had been in an open-label extension study and taking lanthanum carbonate for more than 4 years showed no evidence of low turnover or aluminium-like effects in their bone biopsies. Clinical studies with up to 6 years follow up have demonstrated no hepatotoxic effects related to treatment in terms of liver enzyme changes.

\section{The place of lanthanum in treatment of hyperphosphatemia}

On the basis of its proven efficacy and safety profiles, it is justifiable to consider lanthanum carbonate as a first-line phosphate binder for dialysis patients. However, such decisions are rarely made on solely clinical grounds, and while it is clearly effective without evidence of toxicity, it is also expensive, with three $500 \mathrm{mg}$ lanthanum tablets costing approximately the same as five $800 \mathrm{mg}$ sevelamer pills. However, once the sevelamer prescription increases beyond this number of pills, the dose of lanthanum becomes cheaper at UK prices. Furthermore the dose of lanthanum can be increased without increasing the number of tablets, since it is manufactured in different strengths - 500, 750, and $1000 \mathrm{mg}$ - making it unique among phosphate binders.

Lanthanum carbonate and sevelamer are both significantly more expensive than calcium-based binders. In the light of current financial constraints and the rising cost of medical care, a cost/benefit pharmaco-economic study comparing lanthanum carbonate with other phosphate binders is warranted but is virtually impossible without conducting prospective outcome studies.

The reduced pill burden combined with palatability and tolerability may lead to improvement in patient adherence to therapy, and therefore better phosphate control, although adherence is not solely related to pill burden. Phosphate can be controlled in most patients with a dose between 1500 and $3000 \mathrm{mg}$ daily, so that the prospect of 2-3 tablets daily is real.

In a multicenter, open-label trial, patients on a stable dialysis regimen were screened while receiving phosphate-binder therapy, then entered into a washout phase (Hutchison and Laville 2008). Patients with serum phosphate $>1.78 \mathrm{mmol} / \mathrm{L}$ after washout entered into the main 12-week treatment phase $(\mathrm{N}=367)$, during which they were treated to target (K/DOQI: $1.13-1.78 \mathrm{mmol} / \mathrm{L}$ ) with lanthanum carbonate monotherapy. Mean serum phosphate levels were significantly reduced after 12 weeks of lanthanum carbonate monotherapy vs previous phosphate-binder therapy. The mean number of phosphatebinder tablets being taken per day at screening was 7.6, but during treatment with lanthanum carbonate, most patients were taking doses of up to $3000 \mathrm{mg} /$ day, achievable with $3 \times 1000 \mathrm{mg}$ tablets per day. These findings suggest that lanthanum carbonate monotherapy offers effective control of serum phosphate and, owing to a low tablet burden, may help to simplify the management of hyperphosphatemia in patients with CKD Stage 5.

\section{Conclusion}

Hyperphosphatemia is prevalent in the dialysis population and is considered by many to be an independent risk factor associated with cardiovascular morbidity and mortality. Its control remains a challenging issue for clinical nephrologists because none of the traditional therapeutic approaches appear entirely satisfactory. Dietary restrictions are difficult to follow and standard hemodialysis is inadequate for removal of phosphate, so that the vast majority of dialysis patients require oral phosphate binders. Until recently none of the available agents fulfilled the criteria of an ideal phosphate binder. The introduction of sevelamer hydrochloride and subsequently lanthanum carbonate represents a significant development in phosphate management. Both are nonaluminium, calcium-free agents. Sevelamer achieves effective phosphate lowering and may attenuate progression of vascular calcification in hamodialysis patients. However, its large pill burden and high cost are major disadvantages which have adversely affected treatment compliance and the wider use of sevelamer.

Lanthanum carbonate represents another step on the way to complete phosphate control. Evidence suggests that it is an effective, well tolerated and safe phosphate binder. Lanthanum does not cause hypercalcemia, is effective in reducing calcium $\times$ phosphate product, and may have a positive effect on bone histology. 


\section{Disclosures}

AJH has received research grants from Shire Pharmaceuticals and has a paid consultancy position with Shire. IAM has no conflicts to disclose.

\section{References}

Al-Baaj F, Speake M, Hutchison AJ. 2005. Control of serum phosphate by oral lanthanum carbonate in patients undergoing haemodialysis and continuous ambulatory peritoneal dialysis in a short-term, placebocontrolled study. Nephrol Dial Transplant, 20:775-82.

Bleyer AJ, Burke SK, Dilton M, et al. 1999. A comparison of a calcium free phosphate binder sevelamer hydrochloride with calcium acetate in the treatment of hyperphosphataemia in haemodialysis patients. Am J Kidney Dis, 33:694-701.

Block GA, Klassen PS, Lazarus JM, et al. 2004. Mineral metabolism, mortality and morbidity in maintenance haemodialysis. $\mathrm{J} \mathrm{Am} \mathrm{Soc} \mathrm{Nephrol,}$ 15:2208-18

Block GA, Port FK. 2000. Re-evaluation of risks associated with hyperphosphataemia and hyperparathyroidism in dialysis patients: recommendation for change in management. Am J Kidney Dis, 35:1226-37.

Chertow GM, Burke SK, Raggi P. Treat to Goal Working Group. 2002. Sevelamer attenuates the progression of coronary and aortic calcification in hemodialysis patients. Kidney Int, 62:245-52.

Damment SJ, De Broe ME, D'Haese PC, et al. 2007. Incredulous effects of lanthanum? Toxicol Lett, 168:186-9.

Damment SJ, Pennick M. 2007. Systemic lanthanum is excreted in the bile of rats. Toxicol Lett, 171:69-77.

Delmez J, Slatopolsky E. 1992. Hyperphosphatemia, its consequence and treatment in patients with chronic renal failure. Am J Kidney Dis, 4:313-7.

Delmez JA, Slatopolsky E, Martin KJ, et al. 1982. Mineral, vitamin D and parathyroid hormone in continuous ambulatory peritoneal dialysis. Kidney Int, 21:862-7.

D’Haese PC, Spasovski GB, Sikole A, et al. 2003. A multicenter study on the effects of lanthanum carbonate (Fosrenol) and calcium carbonate on renal bone disease in dialysis patients. Kidney Int Suppl, 85:S73-8.

Finn WF, Joy MS, Haldick GA. 2004. Efficacy and safety of lanthanum carbonate for reduction of serum phosphorus in patients with chronic renal failure receiving hemodialysis. Clin Nephrol, 62:193-201.

Finn WF. SPD 405-307 Lanthanum Study Group. 2006. Lanthanum carbonate versus standard therapy for the treatment of hyperphosphatemia: safety and efficacy in chronic maintenance hemodialysis patients. Clin Nephrol, 65:191-202.

Gonzalez-Revalderia J, Casares M, de Paula M, et al. 2000. Biochemical and haematological changes in low-level aluminium intoxication. Clin Chem Lab Med, 38:221-5.

Goodman WG, Goldin J, Kuizon BD, et al. 2000. Coronary artery calcification in young adults with end-stage renal disease who are undergoing dialysis. $N$ Engl J Med, 342:1478-83.

Goodman WG. 2001. Vascular calcification in chronic renal failure. Lancet, 358:1115-6.

Hutchison AJ, Al-Baaj F. 2005. Lanthanum carbonate for the treatment of hyperphosphatemia in renal failure and dialysis patients. Expert Opin Pharmacother, 6:319-28.

Hutchison AJ, Barnett ME, Krause R, et al. on behalf of the SPD405-309 Lanthanum Study Group. 2008. Long-term efficacy and safety profile of lanthanum carbonate: Results for up to 6 years of treatment. Nephron Clin Pract. In press.
Hutchison AJ, Laville M. on behalf of the SPD405-313 Lanthanum Study Group. 2008. Switching to lanthanum carbonate monotherapy provides effective phosphate control with a low tablet burden. Nephrol Dial Transplant, Jun 24. [Open accesss Epub ahead of print].

Hutchison AJ, Maes B, Vanwalleghem J, et al. 2005. Efficacy, tolerability, and safety of lanthanum carbonate in hyperphosphatemia: a 6-month, randomized, comparative trial versus calcium carbonate. Nephron Clin Pract, 100:c8-c19.

Hutchison AJ, Maes B, Vanwalleghem J, et al. 2006. Long-term efficacy and tolerability of lanthanum carbonate: results from a 3-year study. Nephron Clin Pract, 102:c61-71.

Hutchison AJ, Speake M, Albaaj F. 2004. Reducing high phosphate levels in patients with chronic renal failure undergoing dialysis: a 4 week, dose finding, open-label study with lanthanum carbonate. Nephrol Dial Transplant, 19:1902-6.

Hutchison AJ. 2004. Improving phosphate binder therapy as a way forward. Nephrol Dial Transplant, 19:119-i24.

Joy MS, Finn WF. 2003. Randomized, double-blind, placebo-controlled, dose titration, phase III study assessing the efficacy and tolerability of lanthanum carbonate. a new phosphate for the treatment of hyperphosphataemia. Am J Kidney Dis, 42:96-107.

Knoll O, Kellinghaus H, Betram HP, et al. 1984. Gastrointestinal absorption of aluminium in chronic renal insufficiency. Contrib Nephrol, 38:24-31.

Kopple JD, Coburn JW. 1973. Metabolic studies of low protein diet in uraemia: 11.Calcium, phosphorous, and magnesium. Medicine, 52:597-607.

Lamb EJ, Hodsman A, van Schalkwyk D, et al. 2007. Serum calcium, phosphate, parathyroid hormone, albumin, aluminium and cholesterol achievement on replacement therapy (Chapter 9). Nephrol Dial Transplant, 22(Supp1 7):105-18.

Lowrie EG, Lew NL. 1990. Death risk in haemodialysis patients: the predictive value of commonly measured variables and an evaluation of death rate differences between facilities. Am J Kidney Dis, $15: 458-82$.

Lowrie GE, New NL. 1990. Death risk in haemodialysis patients: the predictive value of commonly measured variables and evaluation of death rate differences between facilities. Am J Kidney Dis, $15: 458-82$.

Mai M, Emmett M, Sheikh M, et al. 1989. Calcium acetate an effective phosphate binder in patients with renal failure. Kidney Int, 36:690-5.

Malluche HH. 2002. Aluminium and bone disease in chronic renal failure. Nephrol Dial Transplant, 17(Supp1 2):21-4.

McIntyre CW. 2007. New developments in the management of hyperphosphatemia in chronic kidney disease. Semin Dial, 20:337-41.

Pennick M, Dennis K, Damment SJ. 2006. Absolute bioavailability and disposition of lanthanum in healthy human subjects administered lanthanum carbonate. J Clin Pharmacol, 46:738-46.

Qunibi WY, Hootkins RE, McDowell LL, et al. 2004. Treatment of hyperphosphatemia in hemodialysis patients: The Calcium Acetate Renagel Evaluation (CARE Study). Kidney Int, 65:1914-26.

Schaefer K, Umlauf E, von Herrath D. 1992. Reduced risk of hypercalcemia for hemodialysis patients by administering calcitriol at night. Am J Kidney Dis, 19:460-4.

Wills MR, Savory J. 1983. Aluminium poisoning: dialysis encephalopathy, osteomalacia and anaemia. Lancet, 2:29-34. 
